\title{
Special issue on frontiers of applied intelligence
}

\author{
He Jiang
}

Published online: 25 October 2013

(C) Springer Science+Business Media New York 2013

This special issue contains 10 papers, which highlight the frontiers of applied intelligence. The themes of the papers cover applications of intelligent systems from various fields of artificial intelligence, such as agent-based systems, pattern recognition, and decision-making techniques.

The first paper, "Detecting Changing Emotions in $\mathrm{Hu}-$ man Speech by Machine and Humans", by Natalie van der Wal and Wojtek Kowalczyk, considers the development of a system that is able to automatically measure changes of a speakers emotional state. The authors achieve this goal by analyzing the speaker's voice, the validation of the system with a controlled experiment, and the visualization of the results. Various features are extracted from speeches, and machine learning algorithms are applied over the selected features, in order to predict the direction of emotion changes in speech.

The second paper, "Dynamic Game with Perfect and Complete Information Based Dynamic Channel Assignment”, by Xiaochen Lai, Quanli Liu, Wei Wang, Likun Li, Simin Lu, and Ying Zhao, deals with the channel assignment strategy within Ad Hoc networks. The authors propose a novel algorithm of channel assignment based on complete and perfect information dynamic game theory. In contrast to traditional algorithms, the throughput and saturation throughput could be improved; meanwhile the packet loss rate and network delay could be reduced.

The third paper, "Detection of JPEG Double Compression and Identification of Smartphone Image Source and Post-capture Manipulation”, by Qingzhong Liu, Peter Cooper, Lei Chen, Hyuk Cho, Zhongxue Chen, Mengyu Qiao, Yuting Su, Mingzhen Wei, and Andrew Sung, focuses

H. Jiang $(\varangle)$

Dalian University of Technology, Dalian, China

e-mail: jianghe@dlut.edu.cn on improving the detection of JPEG double compression in the digital multimedia forensics research field. The authors combine the marginal density and the neighboring joint density features in discrete cosine transform (DCT) domain, to develop a system that is able to detect JPEG double compression. To achieve this, the authors transplant the neighboring joint density features, and merge these features with marginal density features in DCT domain.

The fourth paper, "Mining Non-Redundant Time-Gap Sequential Patterns", by Show-Jane Yen and Yue-Shi Lee, addresses the problem of mining sequential patterns. The authors propose a new algorithm to discover both the sequential patterns and the time interval between any two items in the pattern, by utilizing the information called the time-gap sequential patterns. Both the performance and the scalability of the proposed algorithm are evaluated through extensive empirical study.

The fifth paper, "Optical Character Recognition in Real Environments using Neural Networks and $k$-Nearest Neighbor", by Oliviu Matei, Petrica Pop, and Honoriu Valean, investigates optical character recognition used in the gas- and electricity-meter environment. The authors propose a new process that combines an artificial neural network and the $k$-nearest neighbor algorithm to achieve the objectives. The advantages of the proposed process over the existing systems include the insensitivity to the possible digit rotations, the possibility to work in various conditions, the ability to deduct and use heuristics for character recognition.

The sixth paper, "Utilizing Theory of Mind for Action Selection Applied in the Domain of Fighter Pilot Training", by Mark Hoogendoorn and Robbert-Jan Merk, highlights an intelligent agent system, which is equipped with theory of mind based reasoning capabilities. The approach compares favorably against the existing methods from two perspectives: (1) the ability to handle the expression of certainties, 
and (2) the ability to predict during a substantial number of time steps.

The seventh paper, "Incremental 3D Reconstruction using Bayesian Learning", by Zehuan Yuan and Tong Lu, presents a novel algorithm for 3D reconstruction, which converts incremental $3 \mathrm{D}$ reconstruction to an optimization problem under the Bayesian learning framework. The authors first initialize a 3D patch model using selected key views. Then the result model is incrementally updated by optimizing the geometric and photometric energy terms, whenever a new image is added. The proposed method finally realizes the incremental learning based on the Bayesian framework.

The eighth paper, "Crime Hotspot Mapping Using the Crime Related Factors-A Spatial Data Mining Approach", by Dawei Wang, Wei Ding, Henry Lo, Tomasz Stepinski, Josue Salazar, and Melissa Morabito, focuses on the technique of Hotspot Mapping, which is a hot topic in the analysis of the spatial characteristics of crimes. The authors introduce a new crime hotspot mapping tool-Hotspot Optimization Tool (HOT), which is an application of spatial data mining to hotspot mapping, based on the concept of Geospatial Discriminative Patterns.

The ninth paper, "On Incorporating the Paradigms of Discretization and Bayesian Estimation to Create a New Family of Pursuit Learning Automata", by Xuan Zhang, OleChristoffer Granmo, and B. John Oommen, deals with the convergence speed issue of learning automata. The authors present a new algorithm, i.e., the Discretized Bayesian Pursuit Algorithm (DBPA), which is implemented by linearly discretizing the action probability space of the Bayesian Pursuit Algorithm. Extensive experimental results demonstrate the superiority of DBPA.

The last paper, "A Real-Time Transportation Prediction System", by Haiguang Li, Zhao Li, Robert T. White, and Xindong $\mathrm{Wu}$, presents a real-time transportation prediction system named VTraffic for Vermont Agencies of Transportation. The VTraffic system is a dynamic real-time traffic prediction system, which is able to provide traffic predictions, as well as travel guidance. In order to leverage all available data, the authors propose a data integration strategy to integrate data from heterogeneous data sources.

The papers in this special issue highlight the advanced research of applied intelligence, and present the potential directions of novel applications. We hope that AI researchers will find the papers interesting, and obtain inspiration for their research.

Finally, we wish to take this opportunity to thank all the authors for contributing their work, and to thank the reviewers for their insightful comments. We further wish to express sincere appreciation to Professor Moonis Ali, the Editor-inChief, for his kind support in publishing the special issue, and also to the staff of Applied Intelligence for their kind assistance in the editorial and the publishing process. 